\title{
Elucidation on the Physicochemical Properties of Potential and Clinically Approved Antiviral Drugs: A Search for Effective Therapies against SARS-CoV-2 Infection
}

\author{
Derick Erl P. Sumalapao
}

Department of Epidemiology and Biostatistics, College of Public Health, University of the Philippines Manila, 625 Pedro Gil St., Ermita, Manila 1000, Philippines.

\begin{abstract}
COVID-19 has been confirmed in millions of individuals worldwide, rendering it a global medical emergency. In the absence of vaccines and the unavailability of effective drugs for the SARS-CoV-2 infection, vaccine development is being continuously explored and several antiviral compounds and immunotherapies are currently being investigated. Given the high similarity in genetic identity between SARS-CoV and SARS-CoV-2, the present investigation identified the interaction between the physicochemical properties and the antiviral activity of different potential and clinically approved antiviral drugs against SARS-CoV using hierarchically weighted principal component analysis. Representative drugs from the classes of neuraminidase inhibitors, reverse transcriptase inhibitors, protease inhibitors, nucleoside analogues, and other compounds with potential antiviral activity were examined. The pharmacologic classification and the biological activity of the different antiviral drugs were described using indices, namely, rotatable bond count, molecular weight, heavy atom count, and molecular complexity (92.32\% contribution rate). The physicochemical properties and inhibitory action against SARS-CoV-2 of lopinavir, chloroquine, ivermectin, and ciclesonide validated the adequacy of the current computational approach. The findings of the present study provide additional information, although further investigation is warranted to identify potential targets and establish exact mechanisms, in the emergent search and design of antiviral drug candidates and their subsequent synthesis as effective therapies for COVID-19.
\end{abstract}

Keywords: COVID-19, neuraminidase inhibitors, nucleoside analogues, principal component analysis, protease inhibitors, reverse transcriptase inhibitors

*Correspondence: dpsumalapao1@up.edu.ph

(Received: May 07, 2020; accepted: May 19, 2020)

Citation: Sumalapao DEP. Elucidation on the Physicochemical Properties of Potential and Clinically Approved Antiviral Drugs: A Search for Effective Therapies against SARS-CoV-2 Infection. J Pure Appl Microbiol. 2020;14(suppl 1):1025-1034. doi: 10.22207/ JPAM.14.SPL1.41

(C) The Author(s) 2020. Open Access. This article is distributed under the terms of the Creative Commons Attribution 4.0 International License which permits unrestricted use, sharing, distribution, and reproduction in any medium, provided you give appropriate credit to the original author(s) and the source, provide a link to the Creative Commons license, and indicate if changes were made. 


\section{INTRODUCTION}

SARS-CoV-2, a novel severe acute respiratory syndrome coronavirus 2 , has been identified to cause coronavirus disease 2019 (COVID-19) ${ }^{1}$. COVID-19 is a recent medical emergency worldwide with more than 3.5 million confirmed cases and more than a quarter of a million deaths as of May 4, 2020². Among infected patients, supportive care to help alleviate the symptoms has been recommended ${ }^{3}$. At present, neither effective drugs exist nor vaccines are available for COVID-19; however, vaccines are being developed and several antiviral agents, chemotherapeutics, and immunotherapies are being investigated as pharmacologic interventions.

Despite the very complex process, the search for effective therapies for COVID-19 continues. As SARS-CoV and SARS-CoV-2 have high similarity in genetic identity ${ }^{4,5}$, drugs with inhibitory action against SARS-CoV would exhibit similar degrees of inhibition against SARS-CoV-2. In the present investigation, representative drugs from the classes of neuraminidase inhibitors (NAIs), reverse transcriptase inhibitors (RTIs), protease inhibitors, nucleoside analogues, and other compounds with potential antiviral activity were examined. The chemical and physical properties of drugs such as hydrogen bond donor and acceptor counts, topological polar surface area, heavy atom and rotatable bond counts, complexity, and molecular weight were identified to influence their biological activities ${ }^{6,7}$. Hence, the present study identified the relationship between the physicochemical properties and the antiviral activity against SARS-CoV of the available potential and clinically approved antiviral drugs using hierarchically weighted principal component analysis. Identifying the relationship between the properties of a compound and its biological activity has always been considered important in drug design $^{8}$. The generated relationship will identify significant chemical and physical properties of these antiviral drugs explaining inhibition variations against SARS-CoV-2. The findings of the present investigation offer additional insights relevant to the search for potential antiviral drug candidates and their subsequent synthesis as effective therapies for COVID-19.

\section{MATERIALS AND METHODS}

Chemical and physical properties of drugs with antiviral activity

Information on the potential and clinically approved antiviral drugs against SARSCoV was retrieved from the literature. These are representative drugs from the classes of NAIs, RTIs, protease inhibitors, nucleoside analogues, and compounds with potential antiviral activity. In the class of nucleoside analogues, representative drugs such as acyclovir, foscavir, and ganciclovir were identified. Another major antiviral pharmacologic class examined was the human immunodeficiency virus (HIV) antiretroviral drugs, including indinavir, nelfinavir, and saquinavir as protease inhibitors. For the HIV antiretroviral drugs classified as RTIs, selected HIV nucleoside RTIs included in this study were lamivudine and zidovudine. The fourth pharmacologic class of the antivirals examined was the NAls with oseltamivir and zanamivir as representative drugs. In addition to these clinically approved antiviral drugs, 10 other commercially available drugs with potential antiviral activity were also included. The drugs which exhibited in vitro antiviral activity against SARS-CoV were amantadine ${ }^{9}$, calpain inhibitor III $^{10}$, calpain inhibitor $\mathrm{VI}^{11}$, chloroquine ${ }^{12}$, cinanserin ${ }^{13}$, glycyrrhizin ${ }^{14}$, mizoribine $^{15}$, niclosamide ${ }^{16}$, ribavirin $^{9,10,15}$, and valinomycin ${ }^{17}$. The chemical and physical properties of these compounds, namely topological polar surface area, heavy atom count, hydrogen bond acceptor count, hydrogen bond donor count, complexity, rotatable bond count, and molecular weight were retrieved from online databases $^{18-37}$ (Table 1).

Principal component analysis and computational validation

Similarity in the biological activity and pharmacologic classification of the different potential and clinically approved antiviral drugs were determined, and the presence of correlations among the various chemical and physical properties of these antiviral drugs was identified using principal component analysis. A principal component contains uncorrelated linear combinations of the drug indices with maximum variance, which suggests that a linear transformation must be performed among 
Table 1. Chemical and physical properties of potential and clinically approved antiviral drugs

\begin{tabular}{lrrrrrrr}
\hline Drug & HBDC & HBAC & COMPLEX & HEAVY & ROTA & TOPO & MW \\
\hline Acyclovir $^{18}$ & 3 & 5 & 308 & 16 & 4 & 115.0 & 225.20 \\
Amantadine $^{19}$ & 2 & 1 & 144 & 12 & 0 & 26.0 & 187.71 \\
Calpain Inhibitor III $^{20}$ & 2 & 4 & 497 & 28 & 10 & 84.5 & 382.50 \\
Calpain Inhibitor VI $^{21}$ & 2 & 6 & 537 & 25 & 9 & 101.0 & 372.50 \\
Chloroquine $^{22}$ & 1 & 3 & 309 & 22 & 8 & 28.2 & 319.90 \\
Cinanserin $^{23}$ & 1 & 3 & 391 & 24 & 8 & 57.6 & 340.50 \\
Foscavir $^{24}$ & 0 & 5 & 103 & 10 & 0 & 103.0 & 191.95 \\
Ganciclovir $^{25}$ & 4 & 6 & 346 & 18 & 5 & 135.0 & 255.23 \\
Glycyrrhizin $^{26}$ & 8 & 16 & 1730 & 58 & 7 & 267.0 & 822.90 \\
Indinavir $^{27}$ & 4 & 7 & 952 & 45 & 12 & 118.0 & 613.80 \\
Lamivudine $^{28}$ & 2 & 4 & 331 & 15 & 2 & 113.0 & 229.26 \\
Mizoribine $^{29}$ & 5 & 7 & 329 & 18 & 3 & 151.0 & 259.22 \\
Nelfinavir $^{30}$ & 4 & 6 & 830 & 40 & 10 & 127.0 & 567.80 \\
Niclosamide $^{31}$ & 2 & 4 & 404 & 21 & 2 & 95.2 & 327.12 \\
Oseltamivir $^{32}$ & 2 & 5 & 418 & 22 & 8 & 90.6 & 312.40 \\
Ribavirin $^{33}$ & 4 & 7 & 304 & 17 & 3 & 144.0 & 244.20 \\
Saquinavir $^{34}$ & 5 & 7 & 1140 & 49 & 13 & 167.0 & 670.80 \\
Valinomycin $^{35}$ & 6 & 18 & 1910 & 78 & 9 & 332.0 & 1111.30 \\
Zanamivir $^{36}$ & 7 & 8 & 518 & 23 & 6 & 201.0 & 332.31 \\
Zidovudine $^{37}$ & 2 & 6 & 484 & 19 & 3 & 93.2 & 267.24 \\
& & & & & & & \\
\hline
\end{tabular}

HBDC: hydrogen bond donor count, HBAC: hydrogen bond acceptor count, COMPLEX: complexity, HEAVY: heavy atom count, ROTA: rotatable bond count, TOPO: topological polar surface area $\left(\mathrm{A}^{2}\right), \mathrm{MW}$ : molecular weight $(\mathrm{g} / \mathrm{mol})$

correlated variables, and the linearly transformed variables are subsequently arranged in order of decreasing variances ${ }^{38}$. A principal component is considered for inclusion when it has a loading eigenvalue of at least 1.0. For the retention of the drug property within a principal component, a minimum of 0.30 in absolute value is required as the correlation coefficient between the variable and its principal component. Sampling adequacy was assessed using Kaiser-Meyer-Olkin measure. Numerical calculations and data analysis were performed using STATA ${ }^{\circ}$ V12.0 software. After obtaining the principal components and identifying the significant indices within the components, additional clinically approved antiviral drugs including abacavir ${ }^{39}$, darunavir ${ }^{40}$, didanosine $\mathrm{e}^{41}$, galidesivir ${ }^{42}$, stavudine ${ }^{43}$, and zalcitabine ${ }^{44}$ for pharmacologic classification and some drugs that inhibited SARS-CoV-2 such as chloroquine ${ }^{45}$, lopinavir ${ }^{45}$, ivermectin ${ }^{46}$, and ciclesonide ${ }^{45}$ were utilized to validate the results of the multivariate computational approach.

\section{RESULTS}

Twenty different compounds with potential and clinically approved antiviral activity against SARS-CoV were classified using principal component analysis. Two principal components were obtained with eigenvalues 5.41 and 1.05 . Within a component, the loading values of each drug index were computed (Table 2). The important indices included in the first principal component were complexity $(r=0.4202)$, heavy atom count $(r=0.4123)$, and molecular weight $(r=0.4118)$ with a $77.35 \%$ contribution rate. The number of rotatable bond count $(r=0.7441)$ was the main index in the second principal component, with $14.97 \%$ contribution rate. The rotatable bond count, complexity, heavy atom count, and molecular weight indices primarily defined the pharmacologic classification of the compounds with potential and clinically approved antiviral activity $(92.32 \%$ total contribution rate, 0.7620 Kaiser-Meyer-Olkin sampling adequacy). The main indices in the first principal component, namely complexity, heavy atom count, and 
Sumalapao | J Pure Appl Microbiol | 14(suppl 1):1025-1034 | May 2020 | https://doi.org/10.22207/JPAM.14.SPL1.41

Table 2. Eigenvector loading values, eigenvalues, and measure of sampling adequacy

\begin{tabular}{lccc}
\hline $\begin{array}{l}\text { Antiviral } \\
\text { Property }\end{array}$ & $\begin{array}{c}\text { Component } 1 \\
\text { (e: } 5.4145, \mathrm{p}: 0.7735)\end{array}$ & $\begin{array}{c}\text { Component 2 } \\
\text { (e: } 1.0479, \mathrm{p}: 0.1497)\end{array}$ & $\begin{array}{c}\text { KMO } \\
\text { (Overall: 0.7620) }\end{array}$ \\
\hline MW & 0.4118 & 0.1920 & 0.7286 \\
HBDC & 0.3426 & -0.3493 & 0.7441 \\
HBAC & 0.3986 & -0.2926 & 0.8107 \\
ROTA & 0.2434 & 0.7441 & 0.6416 \\
TOPO & 0.3852 & -0.3843 & 0.7583 \\
HEAVY & 0.4123 & 0.2180 & 0.7183 \\
COMPLEX & 0.4202 & 0.0816 & 0.8754 \\
\hline
\end{tabular}

KMO: Kaiser-Meyer-Olkin measure of sampling adequacy, e: eigenvalue, p: proportion

molecular weight were positively correlated with the rest of the chemical and physical properties of the antiviral drugs (Table 3). Rotatable bond count, the leading index in the second principal component, was positively correlated with heavy atom count, complexity, and molecular weight (Table 3).
The comprehensive scores for the different antiviral drugs were calculated using hierarchical weighted principal component analysis (range: 117.41-1087.30, Table 4). Interestingly, higher comprehensive scores (497.74-644.64) were identified among protease inhibitors (indinavir, nelfinavir, and saquinavir).

Table 3. Correlation between the physicochemical properties of the antiviral drugs

\begin{tabular}{lccccccc}
\hline & MW & HBDC & HBAC & ROTA & TOPO & HEAVY & COMPLEX \\
\hline MW & 1.0000 & $0.6190^{*}$ & $0.8349^{*}$ & $0.6293^{*}$ & $0.7701^{*}$ & $0.9980^{*}$ & $0.9793^{*}$ \\
HBDC & $0.6190^{*}$ & 1.0000 & $0.7752^{*}$ & 0.2937 & $0.8373^{*}$ & $0.6300^{*}$ & $0.7013^{*}$ \\
HBAC & $0.8349^{*}$ & $0.7752^{*}$ & 1.0000 & 0.2284 & $0.9567^{*}$ & $0.8236^{*}$ & $0.8868^{*}$ \\
ROTA & $0.6293^{*}$ & 0.2937 & 0.2824 & 1.0000 & 0.2331 & $0.6613^{*}$ & $0.5679^{*}$ \\
TOPO & $0.7701^{*}$ & $0.8373^{*}$ & $0.9567^{*}$ & 0.2331 & 1.0000 & $0.7598^{*}$ & $0.8214^{*}$ \\
HEAVY & $0.9980^{*}$ & $0.6300^{*}$ & $0.8236^{*}$ & $0.6613^{*}$ & $0.7598^{*}$ & 1.0000 & $0.9789^{*}$ \\
COMPLEX & $0.9793^{*}$ & $0.7013^{*}$ & $0.8868^{*}$ & $0.5679^{*}$ & $0.8214^{*}$ & $0.9789^{*}$ & 1.0000 \\
\hline
\end{tabular}

*significant at $1 \%$

The pharmacologic classes of clinically approved antivirals with high scores next to that of protease inhibitors were NAls (267.02-334.50) and RTIs (214.44-273.51). Among the clinically approved antiviral drugs, the nucleoside analogues had the lowest comprehensive scores (124.11-235.21). Moreover, among the drugs with potential antiviral activity against SARS-CoV, valinomycin and glycyrrhizin had the highest comprehensive scores (1087.30 and 914.51, respectively), whereas amantadine had the lowest comprehensive score (117.41). The comprehensive scores of the calpain inhibitors III and VI (412.73 and 431.33 , respectively) were close to those of the protease inhibitors. Chloroquine and ribavirin had comprehensive scores comparable to those of lamivudine. Cinanserin and mizoribine were comparable with ganciclovir, whereas niclosamide and oseltamivir had similar comprehensive scores. In the validation of the computational approach for pharmacologic classification (Table 5), compounds such as abacavir, didanosine, galidesivir, stavudine, and zalcitabine obtained the lowest comprehensive scores (201.12-259.92). The comprehensive scores of these compounds were within the range of the comprehensive scores for nucleoside analogues and the RTIs (Table 4). Darunavir and lopinavir obtained comprehensive scores similar to those of other protease inhibitors examined in this study. The comprehensive score of ciclesonide was within the range of protease inhibitors, whereas ivermectin scored higher than any of the examined protease inhibitors but was inferior to glycyrrhizin and valinomycin. Moreover, 
Sumalapao | J Pure Appl Microbiol | 14(suppl 1):1025-1034 | May 2020 | https://doi.org/10.22207/JPAM.14.SPL1.41

Table 4. Comprehensive evaluation of the antiviral drugs based on the identified principal components

\begin{tabular}{lcccc}
\hline Drug & Component 1 & Component 2 & Comprehensive Score & Rank \\
\hline Amantadine & 153.85 & -10.69 & 117.41 & 1 \\
Foscavir & 168.12 & -39.58 & 124.11 & 2 \\
Acyclovir & 276.07 & -42.27 & 207.22 & 3 \\
Lamivudine & 285.49 & -42.64 & 214.44 & 4 \\
Chloroquine & 283.05 & -5.23 & 218.15 & 5 \\
Ribavirin & 294.94 & -54.50 & 219.98 & 6 \\
Mizoribine & 315.08 & -57.54 & 235.10 & 7 \\
Ganciclovir & 313.68 & -49.56 & 235.21 & 8 \\
Cinanserin & 338.14 & -16.53 & 259.07 & 9 \\
Niclosamide & 352.08 & -35.80 & 266.97 & 10 \\
Oseltamivir & 350.94 & -29.56 & 267.02 & 11 \\
Zidovudine & 360.24 & -34.28 & 273.51 & 12 \\
Calpain inhibitor III & 412.73 & -25.73 & 315.39 & 13 \\
Calpain inhibitor VI & 431.33 & -32.82 & 328.72 & 14 \\
Zanamivir & 447.00 & -75.22 & 334.50 & 15 \\
Nelfinavir & 651.76 & -42.76 & 497.74 & 16 \\
Indinavir & 720.96 & -37.82 & 552.00 & 17 \\
Saquinavir & 844.30 & -56.25 & 644.64 & 18 \\
Glycyrrhizin & 1201.70 & -100.19 & 914.51 & 19 \\
Valinomycin & 1429.49 & -122.99 & 1087.30 & 20 \\
\hline
\end{tabular}

validation of the statistical methods for inhibitory potency (Table 5) using drugs with established inhibitory action against SARS-CoV-2, revealed

the following comprehensive scores: chloroquine (218.15), lopinavir (553.40), ciclesonide (569.06), and ivermectin (891.27).

Table 5. Physicochemical properties and PCA comprehensive evaluation of potential and clinically approved antiviral drugs

\begin{tabular}{|c|c|c|c|c|c|c|c|c|c|c|}
\hline \multirow[b]{2}{*}{ Drug } & \multirow[b]{2}{*}{ HBDC } & \multirow[b]{2}{*}{ HBAC } & \multirow[b]{2}{*}{ COMPLEX } & \multirow[b]{2}{*}{ HEAVY } & \multirow[b]{2}{*}{ ROTA } & \multirow[b]{2}{*}{ TOPO } & \multirow[b]{2}{*}{ MW } & \multicolumn{3}{|c|}{ Component Scores } \\
\hline & & & & & & & & 1 & 2 & Overall \\
\hline Zalcitabine $^{44}$ & 2 & 3 & 327 & 15 & 2 & 88.0 & 211.22 & 266.43 & -33.11 & 201.12 \\
\hline Didanosine $e^{41}$ & 2 & 5 & 348 & 17 & 2 & 89.0 & 236.23 & 287.36 & -33.30 & 217.29 \\
\hline Chloroquine ${ }^{22}$ & 1 & 3 & 309 & 22 & 8 & 28.2 & 319.90 & 283.05 & -5.23 & 218.15 \\
\hline Stavudine ${ }^{43}$ & 2 & 4 & 388 & 16 & 2 & 79.0 & 224.21 & 294.64 & -29.53 & 223.48 \\
\hline Galidesivir ${ }^{42}$ & 6 & 7 & 334 & 19 & 2 & 140.0 & 265.27 & 316.19 & -54.41 & 236.43 \\
\hline Abacavir ${ }^{39}$ & 3 & 6 & 414 & 21 & 4 & 102.0 & 286.33 & 343.24 & -37.27 & 259.92 \\
\hline Darunavir ${ }^{40}$ & 3 & 9 & 853 & 38 & 12 & 149.0 & 547.70 & 661.65 & -49.38 & 504.39 \\
\hline Lopinavir ${ }^{47}$ & 4 & 5 & 940 & 46 & 15 & 120.0 & 628.80 & 722.48 & -36.35 & 553.40 \\
\hline Ciclesonide ${ }^{48}$ & 1 & 7 & 1100 & 39 & 6 & 99.0 & 540.70 & 742.27 & -33.97 & 569.06 \\
\hline Ivermectin ${ }^{49}$ & 3 & 14 & 1680 & 62 & 8 & 170.0 & 875.10 & 1163.96 & -60.43 & 891.27 \\
\hline
\end{tabular}

PCA: principal component analysis, HBDC: hydrogen bond donor count, HBAC: hydrogen bond acceptor count, COMPLEX: complexity, HEAVY: heavy atom count, ROTA: rotatable bond count, TOPO: topological polar surface area $\left(\mathrm{A}^{2}\right), \mathrm{MW}$ : molecular weight $(\mathrm{g} / \mathrm{mol})$

\section{DISCUSSION}

Among the chemical and physical properties of the potential and clinically approved antiviral drugs evaluated in this study, a positive correlation was identified in all drug properties, including complexity, heavy atom count, and molecular weight. Molecular weight has been considered an important compound property in small drug discovery ${ }^{50}$ and is closely examined in drug optimization steps ${ }^{51}$. In addition to the 
molecular weight in the first principal component, the main index complexity was positively correlated with hydrogen bond acceptor and rotatable bond counts. Molecular complexity, which includes the cardinality of rings, stereocenters, and $s p^{3}$ hybridized carbons, has been related to biological activity $^{52}$. A compound with at least four aromatic rings has high toxicity risks and low compound developability ${ }^{53}$ which justifies the preference for moderately complex structures as lead compounds ${ }^{54}$.

Compounds classified as NAIs, RTIs, protease inhibitors, nucleoside analogues, and some drugs with potential antiviral activity were examined. Among the nucleoside analogues, when acyclovir, foscavir, and ganciclovir were compared, there was an inverse relationship between the calculated comprehensive score and the biological activity $\left(\mathrm{IC}_{50}\right)$ of the antiviral drugs. For instance, when foscavir and acyclovir were examined against herpes simplex virus, acyclovir (IC $50: 0.06 \mu \mathrm{mol} / \mathrm{mL}$ ) was more potent than foscavir $\left(\mathrm{IC}_{50}: 0.44 \mu \mathrm{mol} / \mathrm{mL}\right)^{9}$. The comprehensive score of acyclovir and foscavir was 207.22 and 124.11, respectively. Similarly, ganciclovir $\left(\mathrm{IC}_{50}: 0.014\right.$ $\mu \mathrm{mol} / \mathrm{mL}$ ) was more potent than foscavir (IC $\mathrm{C}_{50}: 0.80$ $\mu \mathrm{mol} / \mathrm{mL}$ ) against cytomegalovirus ${ }^{9}$. Ganciclovir had a higher comprehensive score (235.11) than foscavir (124.11). These observations led to the examination of a possible relationship between the comprehensive score based on physicochemical properties and the potency of the nucleoside analogues against viruses. The higher the comprehensive score of the nucleoside analogue, the more potent was the antiviral drug (lower $\mathrm{IC}_{50}$ ). A nucleoside analogue inhibits viral polymerase and interferes with nucleic acid synthesis ${ }^{18,24,25}$. Acyclovir ${ }^{18}$, foscavir ${ }^{24}$, and ganciclovir ${ }^{25}$ target DNA viruses such as varicellazoster virus and herpes simplex virus. However, acyclovir is more potent than foscavir (Foscarnet) as the former targets herpesvirus and varicellazoster virus polymerases ${ }^{18}$, whereas the latter selectively blocks the pyrophosphate binding site of herpes virus-specific DNA polymerases ${ }^{24}$. Among the nucleoside analogues examined in the present study, ganciclovir was the most potent (the lowest $\mathrm{IC}_{50}$ and the highest comprehensive score). Ganciclovir inhibits replication of several viruses including varicella zoster virus, herpes simplex virus-1 and -2, Epstein-Barr virus, and cytomegalovirus ${ }^{25}$.

Another major antiviral pharmacologic class examined was the protease inhibitor HIV antiretroviral drugs, including indinavir, nelfinavir, and saquinavir. Among these protease inhibitors, saquinavir (Fortovase) had the highest comprehensive score (644.64) and was the most potent $\left(\mathrm{IC}_{50}: 3 \times 10^{-5} \mathrm{\mu mol} / \mathrm{mL}\right)$ against $\mathrm{HIV}^{9}$. Indinavir (Crixivan) was less potent $\left(\mathrm{IC}_{50}: 1 \mathrm{x}\right.$ $10^{-4} \mu \mathrm{mol} / \mathrm{mL}$ ) than saquinavir ${ }^{9}$ and had a lower comprehensive score (552.00). Nelfinavir inhibited SARS-CoV replication in Vero cells ${ }^{55}$, whereas SARSCoV replication in FRhK-4 cells was inhibited by lopinavir ${ }^{56}$. Moreover, lopinavir $\left(\mathrm{IC}_{50}: 4 \mu \mathrm{g} / \mathrm{mL}\right)$ was more potent than ribavirin $\left(\mathrm{IC}_{50}: 50 \mu \mathrm{g} / \mathrm{mL}\right.$ ) against SARS-CoV ${ }^{56}$. Among the HIV antiretroviral drugs classified as RTIs, those examined were lamivudine and zidovudine. Zidovudine $\left(\mathrm{IC}_{50}: 4.86 \times 10^{-5} \mathrm{\mu mol} /\right.$ $\mathrm{mL}$ ) was more potent than lamivudine (Epivir, $\mathrm{IC}_{50}$ : $\left.1.5 \times 10^{-4} \mu \mathrm{mol} / \mathrm{mL}\right)^{9}$. This finding explains why zidovudine had a higher comprehensive score than lamivudine. Zidovudine ${ }^{37}$ has activity against HIV-1, whereas lamivudine ${ }^{28}$ has activity against hepatitis $B$ virus and HIV.

The fourth pharmacologic class of the antivirals examined was the NAls. Representative drugs included were oseltamivir and zanamivir. Zanamivir had a higher comprehensive score than oseltamivir (334.50 vs. 267.02 ), suggesting that zanamivir is more potent than oseltamivir, and this comparison was supported by previous studies. When the two NAls were tested against influenza virus, zanamivir (Relenza) had a lower inhibitory concentration value than oseltamivir (Tamiflu) ${ }^{9}$. In addition, zanamivir ( $\left.\mathrm{IC}_{50}: 2.7 \mathrm{nM}\right)$ was significantly more potent than oseltamivir $\left(\mathrm{IC}_{50}: 8.5 \mathrm{nM}\right)$ when tested against influenza $B$ virus isolate ${ }^{57}$.

In addition to the clinically approved antiviral drugs, 10 other commercially available drugs with potential antiviral activity were also examined. The drugs exhibiting in vitro antiviral activity against SARS-CoV were amantadine ${ }^{9}$, calpain inhibitor $\mathrm{II}^{10}$, calpain inhibitor $\mathrm{VI}^{11}$, chloroquine ${ }^{12}$, cinanserin ${ }^{13}$, glycyrrhizin $^{14}$, mizoribine $^{15}$, niclosamide ${ }^{16}$, ribavirin ${ }^{9,10,15}$, and valinomycin ${ }^{17}$. Among these drugs, amantadine obtained the lowest comprehensive score (117.41). Amantadine (Symmetrel) exhibited antiviral activity against influenza virus $\left(\mathrm{ED}_{50}: 0.1-\right.$ 
25), but did not inhibit, even at $1 \mathrm{mg} / \mathrm{mL}$, SARS-

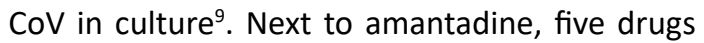
with potential antiviral activities against SARS-CoV had comprehensive scores of less than 300 , and these were chloroquine, cinanserin, mizoribine, niclosamide, and ribavirin. Ribavirin inhibits both DNA and RNA viruses $\left(E_{50}: 1-100 \mu \mathrm{g} / \mathrm{mL}\right)^{9}$. At 5 $\mathrm{mg} / \mathrm{mL}$, ribavirin displayed complete inhibition of SARS-CoV in culture ${ }^{9}$ but cytotoxic on VeroE6 cells $(0.2-1 \mathrm{mg} / \mathrm{mL})^{10}$. Despite ribavirin and mizoribine having comparable comprehensive scores (219.98 and 235.10), neither was recommended as a single treatment agent of SARS ${ }^{15}$.

Recently, chloroquine exhibited inhibitory action against SARS-CoV-2 (IC $\left.\mathrm{I0}_{50}: 9.12 \mu \mathrm{M}\right)^{45}$. Previous study on chloroquine $\left(\mathrm{EC}_{50}: 8.8 \mu \mathrm{M}\right)$ revealed inhibition of SARS-CoV replication in Vero cells $^{12}$. When compared with another antiparasitic drug, niclosamide, inhibition of SARS-CoV replication in Vero cells was likewise observed $\left(\mathrm{EC}_{50}: 2 \mu \mathrm{M}\right)^{16}$. These findings support niclosamide having a higher comprehensive score than chloroquine (266.97 vs. 218.15 ), as niclosamide was more potent than chloroquine. Moreover, drugs including calpain inhibitors $\mathrm{III}^{10}$ and $\mathrm{VI}^{11}$, and cinanserin ${ }^{13}$ have been shown to inhibit SARS$\mathrm{CoV}$ replication. Cinanserin inhibited replication of SARS-CoV via inhibition of the $3 \mathrm{CL}$ protease $\mathrm{e}^{13}$, whereas calpain inhibitor III, a cathepsin-L-specific inhibitor, inhibited cathepsin-L activity $\left(\mathrm{IC}_{50}\right.$ : $2.5 \mathrm{nM})^{10}$. In comparison, glycyrrhizin inhibited SARS-CoV replication, but was difficult to obtain in vivo $\left(\mathrm{EC}_{50}: 300 \mu \mathrm{g} / \mathrm{mL} \sim 365 \mu \mathrm{M}\right)^{14}$, whereas valinomycin, a peptidic insecticide $\mathrm{K}^{+}$-transporter ${ }^{17}$, remained as the most potent inhibitor $\left(\mathrm{EC}_{50}: 0.85\right.$ $\mu \mathrm{M})$ of SARS-CoV replication in Vero cells, and had the highest comprehensive score among the investigated compounds.

Of the ten compounds utilized for validation, six were investigated for the pharmacologic classification and the remaining four for inhibitory potency. Validation of the computational approach for pharmacologic classification revealed low comprehensive scores among abacavir, didanosine, galidesivir, stavudine, and zalcitabine. These comprehensive scores were within the established ranges for nucleoside analogues and RTIs. Interestingly, abacavir, didanosine, and stavudine are nucleoside RTIs $^{39,41,43}$. Abacavir has activity against HIV-1 (HIV-1IIIB EC EC $_{50}$ 3.7-5.8 $\mu \mathrm{M}$ and HIV-1BaL EC ${ }_{50}$ : 0.07-1.0 $\mu \mathrm{M})^{39}$. Moreover, darunavir and lopinavir, both protease inhibitors ${ }^{40,47}$, had comprehensive scores similar to those of other protease inhibitors (indinavir, nelfinavir, and saquinavir). Similar to darunavir, lopinavir also inhibits the activity of an enzyme critical for the HIV viral lifecycle but has a high likelihood of drug interactions ${ }^{47}$.

Despite the scarcity of literature regarding compounds inhibiting SARS-CoV-2, validation of the computational methods revealed congruence between the calculated comprehensive score and the inhibitory potency against SARS-CoV-2 of the following drugs: chloroquine < lopinavir < ciclesonide < ivermectin. Recently, chloroquine $\left(\mathrm{IC}_{50}: 9.12 \mu \mathrm{M}\right)$ and lopinavir $\left(\mathrm{IC}_{50}: 7.28 \mu \mathrm{M}\right)$ were found to inhibit SARS-CoV- $2^{45}$. In comparison, ciclesonide inhibits $\left(\mathrm{IC}_{50}: 4.33 \mu \mathrm{M}\right)^{45}$ and blocks $\left(\mathrm{EC}_{90}: 6.3 \mu \mathrm{M}\right)^{58}$ SARS-CoV-2 replication. Lastly, ivermectin $\left(\mathrm{IC}_{50}: \sim 2 \mu \mathrm{M}\right)$ was effective, with no toxicity observed at all tested concentrations, against SARS-CoV- $2^{46}$.

\section{CONCLUSION}

The chemical and physical properties of potential and clinically approved antiviral drugs explained their pharmacologic classification and biological activity. Hierarchically weighted principal component analysis elucidated the interaction between the physicochemical properties and SARS-CoV inhibition of these antiviral drugs. The physicochemical properties and inhibitory action against SARS-CoV-2 of lopinavir, chloroquine, ivermectin, and ciclesonide validated the adequacy of the current computational approach. The findings of the present study provide additional information, although further investigation is warranted to identify potential targets and establish exact mechanisms, in the emergent search and design of potential antiviral drug candidates and their subsequent synthesis as effective treatment against SARS-CoV-2 infection.

\section{ACKNOWLEDGEMENTS}

None. 


\section{FUNDING}

None.

\section{ETHICS STATEMENTS}

This research is registered with the Research Grants Administration Office, University of the Philippines Manila (RGAO-2020-0448) and does not contain any studies with human participants or animals performed by the author.

\section{DATA AVAILABILITY}

All data analyzed in the study are included in the manuscript and presented as tables.

\section{REFERENCES}

1. Centers for Disease Control and Prevention. 2020. Novel Coronavirus, Wuhan, China, https://www. cdc.gov/coronavirus/2019-ncov/about/index.html (accessed on May 6, 2020)

2. World Health Organization. 2019. Coronavirus disease (COVID-19) Situation Report-105, https:// www.who.int/docs/default-source/coronaviruse/ situation-reports/20200504-covid-19-sitrep-105. pdf?sfvrsn=4cdda8af_2 (accessed on May 6, 2020)

3. Centers for Disease Control and Prevention. 2020. Novel Coronavirus, Wuhan, China: Prevention \& Treatment, https://www.cdc.gov/coronavirus/2019ncov/about/prevention-treatment.html (accessed on May 6, 2020)

4. Hui DS, I Azhar E, Madani TA, et al. The continuing 2019nCoV epidemic threat of novel coronaviruses to global health - The latest 2019 novel coronavirus outbreak in Wuhan, China. Int. J. Infect. Dis. 2020;91:264-266. https://doi.org/10.1016/j.ijid.2020.01.009

5. Zhou P, Yang XL, Wang XG, et al. A pneumonia outbreak associated with a new coronavirus of probable bat 174 origin. Nature. 2020;579(7798):270-273. https://doi. org/10.1038/s41586-020-2012-7.

6. Sumalapao DEP, Gloriani NG. Hierarchically weighted principal component analysis evaluation of the antifungal azoles inhibitory potency on lanosterol 14- $\alpha$-demethylase in Candida albicans. Curr. Res. Environ. Appl. Mycol. 2019;9(1):44-52. https://doi. org/10.5943/cream/9/1/5

7. Sumalapao DEP, Villarante NR, Agapito JD, Asaad AS, Gloriani NG. Topological polar surface area, molecular weight, and rotatable bond count account for the variations in the inhibitory potency of antimycotics against Microsporum canis. J Pure Appl Microbiol. 2020;14(1):247-254. https://doi.org/10.22207/ JPAM.14.1.25

8. Warr WA. Some trends in chem(o)informatics. In: Chemoinformatics and Computational Chemical Biology, Bajorath J. Ed. , Humana Press, New York. 2011; 1-38. https://doi.org/10.1007/978-1-60761839-3_1

9. Tan ELC, Ooi EE, Lin CY, Tan HC, Ling AE, Lim B, Stanton LW. Inhibition of SARS coronavirus infection in vitro with clinically approved antiviral drugs. Emerg Infect. Dis. 2004;10(4):581-586. https://doi.org/10.3201/ eid1004.030458

10. Simmons G, Gosalia DN, Rennekamp AJ, Reeves JD, Diamond SL, Bates P. Inhibitors of cathepsin L prevent severe acute respiratory syndrome coronavirus entry. Proc. Natl Acad Sci. USA. 2005;102(33):11876-11881. https://doi.org/10.1073/pnas.0505577102

11. Barnard DL, Hubbard VD, Burton J, et al. Inhibition of severe acute respiratory syndromeassociated coronavirus (SARSCoV) by calpain inhibitors and $\beta$-DN4-hydroxycytidine. Antivir. Chem. Chemother. 2004;15(1):15-22. https://doi. org/10.1177/095632020401500102

12. Keyaerts E, Vijgen L, Maes P, Neyts J, Van Ranst M. In vitro inhibition of severe acute respiratory syndrome coronavirus by chloroquine. Biochem. Biophys. Res Commun. 2004;323(1):264-268. https://doi. org/10.1016/j.bbrc.2004.08.085

13. Chen L, Gui C, Luo X, et al. Cinanserin is an inhibitor of the $3 \mathrm{C}$-like proteinase of severe acute respiratory syndrome coronavirus and strongly reduces virus replication in vitro. J. Virol. 2005;79(11):7095-7103. https://doi.org/10.1128/jvi.79.11.7095-7103.2005

14. Cinatl J, Morgenstern B, Bauer G, Chandra P, Rabenau $H$, Doerr HW. Glycyrrhizin, an active component of liquorice roots, and replication of SARS-associated coronavirus. Lancet. 2003;361(9374):2045-2046. https://doi.org/10.1016/s0140-6736(03)13615-x

15. Saijo M, Morikawa S, Fukushi S, et al. Inhibitory effect of mizoribine and ribavirin on the replication of severe acute respiratory syndrome (SARS)-associated coronavirus. Antiviral Res. 2005;66(2):159-163. https://doi.org/10.1016/j.antiviral.2005.01.003

16. Wu CJ, Jan JT, Chen CM, et al. Inhibition of severe acute respiratory syndrome coronavirus replication by niclosamide. Antimicrob. Agents Chemother. 2004;48(7):2693-2696. https://doi.org/10.1128/ aac.48.7.2693-2696.2004

17. Wu CY, Jan JT, Ma SH, et al. Small molecules targeting severe acute respiratory syndrome human coronavirus. Proc. Natl Acad Sci. USA. 2004;101(27):10012-10017. https://doi.org/10.1073/pnas.0403596101

18. National Center for Biotechnology Information. PubChem Database. Acyclovir, CID=135398513, https://pubchem.ncbi.nlm.nih.gov/compound/ Acyclovir (accessed on May 4, 2020)

19. National Center for Biotechnology Information. PubChem Database. Amantadine hydrochloride, CID $=64150$, https://pubchem.ncbi.nlm.nih.gov/ compound/Amantadine-hydrochloride (accessed on May 4, 2020)

20. National Center for Biotechnology Information. PubChem Database. CID=9907994, https://pubchem. ncbi.nlm.nih.gov/compound/Carbobenzoxy-valinylphenylalaninal (accessed on May 4, 2020)

21. National Center for Biotechnology Information. PubChem Database. Calpain Inhibitor VI, $C I D=22134166$, https://pubchem.ncbi.nlm.nih.gov/ compound/Calpain-Inhibitor-VI (accessed on May 4, 2020) 
22. National Center for Biotechnology Information. PubChem Database. Chloroquine, $\mathrm{CID}=2719$, https:// pubchem.ncbi.nlm.nih.gov/compound/Chloroquine (accessed on May 4, 2020)

23. National Center for Biotechnology Information. PubChem Database. Cinanserin, CID=5475158, https:// pubchem.ncbi.nlm.nih.gov/compound/Cinanserin (accessed on May 4, 2020)

24. National Center for Biotechnology Information. PubChem Database. Foscarnet sodium, $C I D=44561$, https://pubchem.ncbi.nlm.nih.gov/compound/ Foscarnet-sodium (accessed on May 4, 2020)

25. National Center for Biotechnology Information. PubChem Database. Ganciclovir, CID =135398740, https://pubchem.ncbi.nlm.nih.gov/compound/ Ganciclovir (accessed on May 4, 2020)

26. National Center for Biotechnology Information. PubChem Database. Glycyrrhizin, CID=128229, https:// pubchem.ncbi.nlm.nih.gov/compound/Glycyrrhizin (accessed on May 4, 2020)

27. National Center for Biotechnology Information. PubChem Database. Indinavir, CID=5362440, https:// pubchem.ncbi.nlm.nih.gov/compound/Indinavir (accessed on May 5, 2020)

28. National Center for Biotechnology Information. PubChem Database. Lamivudine, CID=60825, https:// pubchem.ncbi.nlm.nih.gov/compound/Lamivudine (accessed on May 4, 2020)

29. National Center for Biotechnology Information. PubChem Database. Mizoribine, CID=104762, https:// pubchem.ncbi.nlm.nih.gov/compound/Mizoribine (accessed on May 4, 2020)

30. National Center for Biotechnology Information. PubChem Database. Nelfinavir, CID=64143, https:// pubchem.ncbi.nlm.nih.gov/compound/Nelfinavir (accessed on May 4, 2020)

31. National Center for Biotechnology Information. PubChem Database. Niclosamide, CID $=4477$, https:// pubchem.ncbi.nlm.nih.gov/compound/Niclosamide (accessed on May 4, 2020)

32. National Center for Biotechnology Information. PubChem Database. Oseltamivir, CID=65028, https:// pubchem.ncbi.nlm.nih.gov/compound/Oseltamivir (accessed on May 4, 2020)

33. National Center for Biotechnology Information. PubChem Database. Ribavirin, CID=37542, https:// pubchem.ncbi.nlm.nih.gov/compound/Ribavirin (accessed on May 4, 2020)

34. National Center for Biotechnology Information. PubChem Database. Saquinavir, CID=441243, https:// pubchem.ncbi.nlm.nih.gov/compound/Saquinavir (accessed on May 4, 2020)

35. National Center for Biotechnology Information. PubChem Database. Valinomycin, CID=5649, https:// pubchem.ncbi.nlm.nih.gov/compound/Valinomycin (accessed on May 5, 2020)

36. National Center for Biotechnology Information. PubChem Database. Zanamivir, CID=60855, https:// pubchem.ncbi.nlm.nih.gov/compound/Zanamivir (accessed on May 4, 2020)

37. National Center for Biotechnology Information. PubChem Database. Zidovudine, CID=35370, https://
pubchem.ncbi.nlm.nih.gov/compound/Zidovudine (accessed on May 4, 2020)

38. Hastie T, Tibshirani R, Friedman J. Principal components. In: The elements of statistical learning. 2 nd edition. Springer, 2009; 534-541. https://doi. org/10.1007/978-0-387-84858-7

39. National Center for Biotechnology Information. PubChem Database. Abacavir, CID=441300, https:// pubchem.ncbi.nlm.nih.gov/compound/Abacavir (accessed on May 6, 2020)

40. National Center for Biotechnology Information. PubChem Database. Darunavir, CID=213039, https:// pubchem.ncbi.nlm.nih.gov/compound/Darunavir (accessed on May 6, 2020)

41. National Center for Biotechnology Information. PubChem Database. Didanosine, CID $=135398739$, https://pubchem.ncbi.nlm.nih.gov/compound/ Didanosine (accessed on May 6, 2020)

42. National Center for Biotechnology Information. PubChem Database. Galidesivir, CID =10445549, https://pubchem.ncbi.nlm.nih.gov/compound/ Galidesivir (accessed on May 6, 2020)

43. National Center for Biotechnology Information. PubChem Database. Stavudine, CID=18283, https:// pubchem.ncbi.nlm.nih.gov/compound/Stavudine (accessed on May 6, 2020)

44. National Center for Biotechnology Information. PubChem Database. Zalcitabine, CID=24066, https:// pubchem.ncbi.nlm.nih.gov/compound/Zalcitabine (accessed on May 6, 2020)

45. Jeon S, Ko M, Lee J, Choi I, Byun SY, Park S, Shum D, Kim S. Identification of antiviral drug candidates against SARS-CoV-2 from FDA-approved drugs. Antimicrob. Agents Chemother. (accessed on May 4, 2020) doi: 10.1128/AAC.00819-20

46. Caly L, Druce JD, Catton MG, Jans DA, Wagstaff KM. The FDA-approved drug ivermectin inhibits the replication of SARS-CoV-2 in vitro. Antiviral Research. 2020;178:104787. https://doi.org/10.1016/j. antiviral.2020.104787

47. National Center for Biotechnology Information. PubChem Database. Lopinavir, CID=92727, https:// pubchem.ncbi.nlm.nih.gov/compound/Lopinavir (accessed on May 6, 2020)

48. National Center for Biotechnology Information. PubChem Database. Ciclesonide, $C I D=6918155$, https://pubchem.ncbi.nlm.nih.gov/compound/ Ciclesonide (accessed on May 6, 2020)

49. National Center for Biotechnology Information. PubChem Database. Ivermectin, CID=6321424, https:// pubchem.ncbi.nlm.nih.gov/compound/Ivermectin (accessed on May 6, 2020)

50. Meanwell NA. Improving drug candidates by design: a focus on physicochemical properties as a means of improving compound disposition and safety. Chem Res. Toxicol. 2011;24(9):1420-1456. https://doi. org/10.1021/tx200211v

51. Gleeson MP. Generation of a set of simple, interpretable ADMET rules of thumb. J Med Chem. 2008;51(4):817834. https://doi.org/10.1021/jm701122q

52. Hann MM, Oprea TI. Pursuing the lead likeness concept in pharmaceutical research. Curr Opin Chem. 
Biol. 2004;8(3):225-263. https://doi.org/10.1016/j. cbpa.2004.04.003

53. Lovering F, Bikker J, Humblet C. Escape from flatland: increasing saturation as an approach to improving clinical success. J. Med. Chem. 2009;52(21):6752-6756. https://doi.org/10.1021/jm901241e

54. Hann MM, Leach AR, Harper G. Molecular complexity and its impact on the probability of finding leads for drug discovery. J Chem Inf Comput Sci. 2001;41(3):856864. https://doi.org/10.1021/ci000403i

55. Yamamoto N, Yang R, Yoshinaka Y, et al. HIV protease inhibitor nelfinavir inhibits replication of SARSassociated coronavirus. Biochem Biophys Res Commun. 2004;318(3):719-725. https://doi.org/10.1016/j. bbrc.2004.04.083
56. Chu C, Cheng V, Hung I, et al. Role of lopinavir/ritonavir in the treatment of SARS: initial virological and clinical findings. Thorax. 2004;59(3):252-256. https://doi. org/10.1136/thorax.2003.012658

57. Gubareva LV, Webster RG, Hayden FG. Comparison of the activities of zanamivir, oseltamivir, and RWJ270201 against clinical isolates of influenza virus and neuraminidase inhibitor-resistant variants. Antimicrob Agents Chemother. 2001;45(12):3403-3408. https:// doi.org/10.1128/aac.45.12.3403-3408.2001

58. Matsuyama S, Kawase M, Nao N, Shirato K, et al. The inhaled corticosteroid ciclesonide blocks coronavirus RNA replication by targeting viral NSP15. bioRxiv. 2020.03.11.987016. https://doi. org/10.1101/2020.03.11.987016 